\title{
Optimización de los procesos de gestión en cirugía electiva
}

\section{Optimize the management of the operation rooms}

\author{
Ruth Kohnenkampf MD. ${ }^{1, *}$, Cristian Rocco MD. ${ }^{2}$, Bárbara Ortega $^{3}$ \\ Médico Anestesiólogo, Jefa de Unidad de Anestesia y Pabellones Quirúrgicos, Hospital de Quilpué. \\ Médico Anestesiólogo, Clínica Las Condes, Magíster seguridad del paciente gestión y calidad de atención. \\ Enfermera Supervisora Pabellones Quirúrgicos, Hospital de Quilpué.
}

Fecha de recepción: 15 de abril de 2021 / Fecha de aceptación: 21 de abril de 2021

\begin{abstract}
The ward chiefs are team leaders and, as such, they must have the ability to adequately manage their unit in such a way as to meet the real needs of the population they are responsible for serving. To achieve this, it is essential to optimize the management of operating rooms, oriented to a strategic management of resources, which allows the best performance and productivity. In this document we will provide you with some tools, such as: definitions of concepts, analysis of the sub-processes that make up the surgical process and suggestions to improve the management of each of its parts.
\end{abstract}

Key words: Operating room management.

\section{RESUMEN}

Los jefes de pabellón son líderes de equipo y, como tales, deben tener la capacidad de gestionar adecuadamente su unidad, de tal modo de satisfacer las necesidades reales de la población que le corresponde atender. Para lograr esto, es fundamental optimizar el manejo de los quirófanos, orientado a una gestión estratégica de recursos, que permita el mejor rendimiento y productividad. En este documento les entregaremos algunas herramientas, tales como: definiciones de conceptos, análisis de los subprocesos que componen el proceso quirúrgico y sugerencias para mejorar la gestión de cada una de sus partes.

Palabras clave: Gestión de quirófano.

\section{Introducción}

a comisión nacional de productividad en enero de 2020 publicó un informe Ilamado: "Uso Eficiente de Quirófanos Electivos y Gestión de Lista de Espera Quirúrgica No GES"[1], en el que se describe que en Chile actualmente no tenemos la capacidad de resolución de los problemas de salud de la población y, dentro de ese contexto, destacan las patologías con resolución quirúrgica, tanto en el número de pacientes que se encuentran en lista de espera de las Garantías Explícitas en Salud (GES) y no GES, como en los tiempos de espera, sobre todo en el último grupo mencionado que, según el informe, corresponden al $99 \%$ de los casos en lista de espera.

¿Cuál es el objetivo de una óptima gestión de los pabellones quirúrgicos? Conseguir una ocupación máxima del quirófano, con el mayor grado de satisfacción del paciente, el menor costo económico, además de la mayor calidad y seguridad en la cirugía realizada, que implica la menor cantidad de complicaciones asociadas. El pabellón quirúrgico genera alrededor del $40 \%$ o más de los ingresos de un hospital[2\}, por tanto, es primordial hacer un uso eficiente de sus recursos (sobre todo en la realidad actual de implementación del modelo de pago GRD-PERC o Grupos Relacionados por el Diagnóstico-Producción, Eficiencia, Recursos y Costos) para poder aportar recursos para el funcionamiento global del hospital al que pertenece.

El área de pabellón quirúrgico es una de las más difíciles de administrar en el ámbito de la salud, ya que cuenta con muchas variables y puntos de encuentro de diferentes procesos que son indispensables, ninguna puede fallar, porque inevitablemente llevan a un quiebre en la continuidad de la atención. Además, es 
uno de los servicios que causa mayores gastos en una institución de salud, con recursos físicos y humanos limitados, siendo una causa de gran congestión en el funcionamiento de otros servicios hospitalarios y ambulatorios, por lo que es indispensable obtener de ellos el máximo rendimiento[3].

Queda mucho trabajo pendiente por hacer en nuestro país: mientras un quirófano electivo en Chile realiza alrededor de 750 cirugías al año, los referentes internacionales hacen sobre 1.000[1]. Esto refleja lo fundamental que es un manejo racional y programado de los pabellones en todas las áreas que son determinantes en el proceso quirúrgico (pre, intra y posoperatorio) y el gran trabajo que tenemos todos los funcionarios que trabajamos en cargos de coordinación de estas unidades.

El objetivo de este trabajo es entregar herramientas para mejorar el uso de los recursos asociados a los pabellones electivos, con la finalidad de aumentar la oferta de resolución quirúrgica para los casos tanto GES como No GES y disminuir con ello los tiempos de lista de espera quirúrgica, para ofrecer una atención más transparente, oportuna, segura y justa.

\section{Fundamento}

Los hospitales públicos en Chile presentan problemas comunes en la gestión de sus pabellones, lo que se analizó en detalle en el informe de la Comisión Nacional de productividad ya mencionado[1]. El principal problema es una lista de espera en donde un gran número de pacientes llevan esperando más del tiempo definido como máximo de espera, asociado a su diagnóstico médico. Esto podría aumentar las complicaciones perioperatorias en pacientes en donde la cirugía se realizó en forma tardía e incluso a la muerte de aquellos que no pudieron resolver su patología quirúrgica, además de aumentar la insatisfacción y la desconfianza en el sistema[2]. Otro problema muy importante es que no disponemos de un lenguaje único en relación a conceptos básicos del funcionamiento de las unidades de anestesia y pabellón, lo que dificulta el análisis y la utilización de indicadores o protocolos en forma adecuada. Se incluye un glosario de definiciones, en el anexo 1, que el Departamento de Estadística e Información de Salud del Ministerio de Salud (MINSAL) entrega cada año en los manuales REM (resumen estadístico mensual)[4].

\section{¿Cómo logramos obtener la mayor satisfacción de los pa- cientes considerando los limitados recursos disponibles?}

Pilares de esta labor de gestión:

1. Procesos centrados en los pacientes.

2. Mejoría continua de los procesos.

3. Optimizar el uso del recurso pabellón (tasa de ocupación).

4. Reducción de tiempos de listas de espera.

5. Uso eficiente de los recursos humanos.

6. Reducción de los tiempos entre cada intervención (tiempos muertos, atrasos, etc.) y de preparación de los pacientes.

7. Reducción de costos.

8. Aumento de ingreso de recursos económicos para el recinto de salud.

9. Aumentar el número total de cirugías.

10. Disminuir suspensiones.

11. Uso racional de camas, que se relaciona con la capacidad de ambulatorización y trabajo en conjunto con hospitalización domiciliaria.

12. Atención segura, de calidad y equitativa para la población.

13. Implementación y cumplimiento de indicadores de calidad sobre el umbral deseado (por ejemplo, en el adecuado llenado de hojas de evaluación preanestésica u hoja de anestesia).

14. Recurso humano: evaluación continua de cantidad, capacitación y motivación[5].

Estos pilares nos darán resultados que podemos evaluar en indicadores, los cuales nos permiten estimar la eficiencia del uso de los pabellones de 3 maneras[1]:

1. Tasa de ocupación: Los tiempos utilizados de cada quirófano para la realización de cirugías electivas dentro del horario asignado de funcionamiento (horario institucional) se evalúan formalmente con el índice o tasa de ocupación, que es un indicador que se define como relación entre el tiempo disponible y el tiempo real en el que se encuentra ocupado por algún paciente. La tasa de ocupación objetivo de cada institución es discrecional, ya que son muchas las variables que determinan a este indicador. Existen recomendaciones en la literatura, por ejemplo, en el Reino Unido la tasa de utilización es de $80 \%$, en EE. UU. de $75 \%$, en Canadá de $70 \%$ y en Australia entre 75\%-87\%[1]. Se asume que, entre mayor ocupación, mayor producción y aprovechamiento de los recursos[3] lo cual no es directamente proporcional, a mayor ocupación en el mismo tiempo ofertado hay un punto en que se estresa el sistema y aparecen más eventos adversos asociado al "apuro"[3]. Por tanto, obtener y fijar una tasa de ocupación "saludable" es prioritario para la planificación.

2. Cantidad de cirugías electivas: Realizadas por quirófano al día, mensual o al año, como medida de la capacidad de resolución de la demanda. El número de pacientes intervenidos quirúrgicamente está estrechamente relacionado con la disponibilidad de recursos y la programación de la tabla quirúrgica[2]. Se debe relacionar la cantidad de pacientes a las intervenciones realizadas en cada paciente y la duración de la intervención, para tener un diagnóstico del tipo de paciente y cirugías que se realizan para optimizar los recursos.

3. Uso de las horas disponibles y el cumplimiento de la programación: En este punto es necesario analizar la hora de inicio de cada cirugía y el tiempo de retraso. En relación a realizar las cirugías efectivamente programadas aparece como indicador el porcentaje de suspensión. Disminuir la tasa de cirugías programadas no efectuadas mejora en todo aspecto la productividad del pabellón. Y como en casi todos los ámbitos quirúrgicos, podemos tener injerencia en 3 elementos para mejorar esta variable:

- Paciente: ¿Quién es mi paciente? Suena fácil, pero es lo más complejo. Implica que el equipo que maneja los subprocesos pre e intraoperatorios (médicos, enfermeras y técnicos) conozca al paciente como persona, individual, en su aspectos físicos y sicológicos: ¿Tiene algún antecedente mórbido que deba ser compensado o que requiera alguna preparación especial de pabellón? ¿Está en conocimiento de todas las implicancias de la cirugía y está de acuerdo con ellas? ¿Necesita más estudio de especialidad o de exámenes? ¿Debo 
suspenderle algún medicamento o su alimentación? ¿Está claro el diagnóstico? ¿Tiene red de apoyo y las condiciones básicas para los cuidados posoperatorios en su domicilio? Cualquiera de esas preguntas que no pueda ser respondida a cabalidad, puede llevar inevitablemente a una suspensión y, con ello, a la pérdida de todos los recursos económicos y humanos asociados.

- Recurso humano: ¿Disponemos de equipo completo de médicos, enfermeros, técnicos de enfermería de nivel superior (tens) y auxiliares de aseo para poder manejar el pre, intra y posoperatorio del paciente? ¿Contamos con equipos completos en los servicios de apoyo, tales como esterilización o banco de sangre?

- Insumos e infraestructura: ¿Tenemos pabellones y recuperación habilitados para trabajar en forma segura? ¿Disponemos de camas e insumos para todo el proceso quirúrgico en caso de que haya o no haya complicaciones? (instrumental, fármacos, insumos médicos, etc.).

Las suspensiones tienen un componente claramente económico (por la pérdida de recursos humanos y posibilidad de utilizar pabellón), sanitario (porque posterga una cirugía que tiene como objetivo resolver un problema de salud concreto), pero además, tiene una carga afectiva muy importante en el paciente y su red de apoyo: cuando ingresa al hospital ese paciente tiene la expectativa de resolver el problema que lo aqueja, lo cual no se cumplirá y muchas veces causa rabia/frustración. Además, de haber cumplido con ayuno, suspensión de medicamentos, incumplimiento laboral, coordinación de la logística de su hogar y familia por uno o varios días, gasto en medio de transporte, riesgo de adquirir una infección asociada a atención de salud en el proceso (muy importante, sobre todo en época de pandemia), etc. Esto aumenta la insatisfacción usuaria y los reclamos en la OIRS (oficina de información, reclamos y sugerencias).

Analizar las causas de suspensión (paciente, equipo médico, materiales, etc.), nos podrá orientar en los planes de mejora (confirmaciones de tabla anticipada, petición de materiales para compra, etc.). Obtener, fijar y analizar una tasa de suspensión "saludable" es prioritario para la planificación.

\section{Y entonces, ¿cómo logro cambios positivos en mi servicio clínico?}

Analicemos ahora propuestas concretas y prácticas para optimizar el trabajo administrativo en pabellón. Pero ya que es una tarea gigante (por la complejidad intrínseca del proceso quirúrgico), lo fraccionaremos y analizaremos mediante el enfoque de gestión por procesos, para profundizar en los aspectos de calidad de ejecución de los procesos, permitiendo sustentar la acreditación y profundizar el reconocimiento de la red de salud. Esto lo haremos basándonos en un documento del Ministerio de Salud: "Proceso Quirúrgico"[6] que fue desarrollado por la Consultoría para Estandarización de Procesos de Redes Asistenciales en el año 2011 su primera versión y en el año 2015 actualizado. El objetivo del MINSAL fue descomponer la globalidad de cada proceso en flujos de actividades conectadas entre sí, simplificando la variabilidad que aparece en cada establecimiento de salud cuando se prestan los servicios de atención clínica, dando visibilidad a las oportunidades de mejoras que se puedan implementar.

Definición de proceso quirúrgico[7] comprende el conjunto de actividades asistenciales y no asistenciales centradas en el paciente con una indicación quirúrgica, destinadas a la resolución integral del procedimiento indicado, garantizando la continuidad asistencial, calidad y seguridad clínica, confidencialidad e información durante el mismo.

El proceso completo de atención quirúrgica tiene varias partes que lo componen, cada una de ellas con la posibilidad de mejorar su rendimiento. Dependiendo de la fuente, se puede clasificar en 3 o 4 componentes:

1. Proceso diagnóstico e indicación médica.

2. Proceso prequirúrgico (exámenes, evaluación, seguimiento y admisión).

3. Intervención quirúrgica.

4. Proceso posquirúrgico (estancia y egreso).

De estos 4 elementos, los últimos 3 tienen relación directa con el trabajo del equipo administrativo/clínico que gestiona pabellón, por tanto, son en los que nos enfocamos para lograr cambios. De hecho, el Minsal en el año 2015 explicita que dentro del proceso quirúrgico se identifican tres subprocesos: Pre quirúrgico, quirúrgico y posquirúrgico[6], incorporando el diagnóstico e indicación médica como parte del proceso prequirúrgico. Operatoriamente esta clasificación es más adecuada para la gestión de servicios quirúrgicos.

Otra forma de hacer el análisis es a través de los microprocesos que componen el proceso quirúrgico, que desglosaremos en la siguiente Tabla 1 (basados en descripción Minsal)(6):

\section{En relación al subproceso prequirúrgico:}

El Minsal[6] refiere que comienza cuando se genera una solicitud de interconsulta hacia el nivel ambulatorio de especialidades para la resolución de una patología quirúrgica y describe 3 etapas:

\begin{tabular}{|c|c|c|c|c|c|}
\hline Proceso quirúrgico & Priorización & Definición & Preparación & Ejecución & Recuperación \\
\hline Flujo administrativo & & Gestión de recursos & $\begin{array}{l}\text { Preparación de paciente } \\
\text { y de pabellón }\end{array}$ & $\begin{array}{l}\text { Registro de prestaciones } \\
\text { e insumos }\end{array}$ & \\
\hline Flujo clínico & $\begin{array}{l}\text { Priorización de } \\
\text { solicitudes }\end{array}$ & & $\begin{array}{l}\text { Preparación de paciente } \\
\text { y de pabellón }\end{array}$ & Intervención quirúrgica & $\begin{array}{l}\text { Cuidados post } \\
\text { anestesia }\end{array}$ \\
\hline \multicolumn{6}{|l|}{$\begin{array}{l}\text { Atención clínica } \\
\text { hospitalizado }\end{array}$} \\
\hline Flujo operacional & & & $\begin{array}{l}\text { Preparación de paciente } \\
\text { y de pabellón }\end{array}$ & & Gestión de camas \\
\hline
\end{tabular}


- Etapa de gestión de la interconsulta y a su respectivo agendamiento.

- Evaluación clínica del paciente, con o sin indicación de exámenes.

- Ingreso a la lista de espera.

Problemas del subproceso prequirúrgico[4]:

- Pérdida de la trazabilidad del paciente en este proceso.

- Demora en el estudio prequirúrgico de pacientes de cirugía.

- Alto número de días de hospitalización prequirúrgico.

- Alto número de suspensión de cirugías, por inadecuada preparación del paciente, ocasionando subutilización de horas de pabellón.

- Falta de coordinación con la unidad de gestión de la demanda.

- Vencimiento de garantías GES quirúrgicas.

Ya habiendo definido el proceso prequirúrgico y sus problemas asociados, analizaremos los elementos que lo componen y propondremos planes de mejora para ser adaptados a la realidad local de cada institución y poder así ser incorporados en sus protocolos:

1. Programación: Es un procedimiento que es determinante en la producción quirúrgica, que contiene la asignación de pabellones, planificación de los recursos y el orden de intervención de los pacientes. El producto que se obtiene de este proceso corresponde a la tabla quirúrgica[2].

Esta programación es muy compleja, ya que depende de múltiples variables, entre las cuales destacan: la variedad de intervenciones quirúrgicas, las variaciones de los tiempos de intervención (que pueden variar incluso dependiendo del cirujano y que es mayor en centros docentes), las prioridades de los pacientes, las restricciones de la capacidad del centro de salud, equipos médicos utilizados (duplicidad de peticiones en mismo horario), distribución del personal para atender la capacidad de quirófanos (licencias médicas, vacaciones, permisos, etc.), tiempos de recambio de quirófano (aseo y preparación), la ambulatoriedad de algunas intervenciones, los horarios médicos, criterios médicos de asignación y la edad de los pacientes[2],[8]. Además, no todas las intervenciones pueden ser asignadas a todos los médicos, ni a todos los pabellones, ni en todas las jornadas, ni ocupar los mismos insumos.

Por todo lo anterior, la programación de la tabla quirúrgica se vuelve un elemento crucial en la optimización de recursos. Los elementos en que podemos actuar directamente son:

a) Selección de pacientes según lista de espera.

b) Preparación de pacientes en el preoperatorio para que ingresen a la institución de salud en sus condiciones óptimas según cada comorbilidad asociada que presenten y también para que dispongan de toda la información relacionada al perioperatorio (beneficios, riesgos, logística, etc.). Este punto es fundamental para evitar suspensiones.

c) Disponibilidad de recursos necesarios para el perioperatorio: insumos, instrumental, equipos médicos, camas según la necesidad de cada paciente, laboratorio, banco de sangre, fármacos, recursos humanos, etc.

d) Distribución de los tiempos de bloques quirúrgicos designados según especialidad. Usualmente esto se resuelve según una construcción histórica de cada institución o por solicitud de cada equipo quirúrgico, pero lo más justo sería realizar una repartición dinámica según las necesidades reales de cada servicio.

Para realizar una programación óptima, no podemos basarnos en suposiciones sobre la duración de determinadas intervenciones. Es necesario conocer nuestros tiempos reales según los datos históricos de nuestro hospital y no en centros externos, porque existe una gran variabilidad.

2. Formación de Comité Quirúrgico multidisciplinario, con un flujo de comunicación permanente, compuesto por representantes de cada servicio que compone el proceso quirúrgico completo. El Minsal en su documento del proceso prequirúrgico[7] plantea que debe haber una reunión de tabla quirúrgica liderada por el subdirector médico o a quien él delegue, en donde concurran todos los involucrados en la toma de decisiones del proceso, haciendo una tabla quirúrgica idealmente con una semana de anticipación y una definitiva a lo menos 24-28 h antes de la cirugía. Estas reuniones deben ser diarias, acotadas y resolutivas. Esto da dinamismo al esquema de trabajo, para modificar y optimizar la programación según la disponibilidad de recursos, disminuyendo así la posibilidad de suspensión. Por ejemplo, si hay un especialista de vacaciones que no permite dar uso a su bloque de pabellón, se avisa al comité y se redistribuye con un plazo de tiempo adecuado para no dejar sin uso horas de pabellón. O si falta instrumental estéril para realizar una cirugía, el equipo de esterilización alerta modifica la programación, lo mismo si faltan camas en la unidad de cirugía ambulatoria, etc. Implica trabajo en equipo con dedicación, flexibilidad y buena voluntad, pero que mejora en forma efectiva los resultados. Además, el comité quirúrgico estará encargado de la programación semanal de los bloques quirúrgicos por especialidad.

El Minsal refiere que los puntos a evaluar en las reuniones diarias son:

- Causas de suspensión de cirugías programadas el día anterior, para realizar correcciones inmediatas.

- Confirmación de los pacientes propuestos para tabla.

- Revisión de requerimientos especiales para cada caso y confirmación de su disponibilidad.

- Realizar priorización de pacientes frente a contingencias.

- Priorizar pacientes que fueron suspendidos días previos.

- Nosotros planteamos, además, priorizar pacientes hospitalizados en espera de cirugías o procedimientos, para optimizar la gestión de camas.

3. Listas de espera: su adecuado manejo es primordial, no solamente bajo el punto de vista de la salud del paciente individual, sino que a nivel colectivo. Es una forma de objetivar la oferta que ofrece el sistema y la demanda de la población. La resolución de la lista de espera depende, más que del número en sí de pacientes incluidos, de la asignación de recursos, y no tanto de su aumento directo sino de su correcta y adecuada gestión[3]

Una de las formas de mejorar el manejo de los escasos recursos disponibles, es con una adecuada gestión de los tiempos de lista de espera quirúrgicos, lo cual permite al sistema trabajar de una manera más eficiente, mientras que a las personas les permite recibir una atención oportuna que se traduce en una mejor recuperación y calidad de vida. En este punto es clave la programación, punto ya analizado previamente. 
4. Estandarizar criterios de preparación preoperatoria: Implementar un "Protocolo de Preparación Preoperatoria" desarrollado tanto por el equipo médico como de enfermería, es decir, trabajo interdisciplinario. Consideramos que el optimizar las condiciones previo a la cirugía de los pacientes disminuye el riesgo de complicaciones perioperatorias y de suspensión. Esta reducción se traduce en menor morbimortalidad asociada, menos ocupación de camas críticas, menor estancia media hospitalaria, menos costos y, dado todo lo anterior, mayor posibilidad de producción quirúrgica. Este protocolo debe tener en su alcance a todo el personal que tenga injerencia en la etapa preoperatoria del paciente: cirujanos tratantes, equipo prequirúrgico, enfermeras y tens de sala, anestesistas, etc. Debe ser consensuado y aceptado por todos, adecuado a la realidad local. Con los recursos de comunicación digital actual, se puede hacer una difusión rápida y efectiva del material, que esté disponible para revisar en los teléfonos de todo el personal de salud. En anexo 2 se puede ver un ejemplo de tríptico que resume lo fundamental que se debe saber para la preparación de un paciente que se va a operar (derivación a especialistas, exámenes, suspensión de medicamentos). Nuestra recomendación es que cada hospital haga uno propio según su realidad y necesidades.

5. Potenciar el policlínico de anestesia: Cuando no existe policlínico de anestesia o sus horas son escasas, la derivación se hace a medicina interna, broncopulmonar, cardiología u otras especialidades en forma directa, que presentan listas de espera largas en general. Por tanto, la condición inicial o el diagnóstico quirúrgico que llevó a la interconsulta empeora su condición en los meses o incluso años de espera, lo que aumenta el riesgo de complicaciones perioperatorias y, con ello, la estadía hospitalaria. Además, cuando es el tratante quien deriva sin protocolo, suele pedir más exámenes e interconsultas de las necesarias. Es por todo lo anterior que recomendamos el centralizar en el policlínico de anestesia todas las interconsultas prequirúrgicas y que el anestesista defina una real necesidad de derivar a otras especialidades para optimizar las condiciones basales del paciente o de tomar exámenes específicos.

6. Participación del comité quirúrgico en gestión de camas y de salas destinadas a posoperatorio de pacientes: en el informe nacional[1] se describe una brecha de hasta 12 días para mismos de pacientes con similares procedimientos, diagnósticos y factores de riesgo. Unificar criterios para priorización de uso de camas, de altas y de asignación de pabellón para pacientes hospitalizados puede optimizar el uso de los recursos disponibles. La decisión pasa de ser completamente del jefe de servicio a un comité multidisciplinario que puede apoyar en la toma de decisiones. Ejemplo de ello, es trabajar en forma dinámica con equipo de cirugía mayor ambulatoria, salas de hospitalización y unidades de paciente crítico para programar una tabla que se pueda realizar porque tiene los recursos efectivos para hacerlo y que no se suspenda si era evitable.

7. Transparentar información: En este punto podemos caracterizar su problemática en el tipo de datos analizados y sus receptores. Por un lado, los análisis de indicadores son principalmente estadísticos financieros, no orientados a la gestión clínica (son datos agregados que no permiten el seguimiento de los pacientes ni tomar decisiones médicas en base a ellos). Es difícil ejercer mecanismos de control sobre la gestión de los pabellones dada la escasez de datos de relevancia clínica y el acceso a la información[2]. Actualmente, la información de la producción de cada servicio quirúrgico llega a los jefes de pabellón, enfermeras supervisoras y directivos. Estos datos son: número de intervenciones programadas, el número de intervenciones urgentes, el número de intervenciones suspendidas y el índice de ocupación del o los quirófanos. Creemos que incorporar además datos tales como el retraso en el comienzo de la cirugía, el tiempo medio entre intervenciones y el retraso en la hora de finalización de la tabla quirúrgica podrían incorporarse como estadística para poder tomar decisiones que puedan optimizar dichos indicadores. Sugerimos ampliar el espectro de funcionarios que reciben la información de productividad mensual/anual, dado que los clínicos de todos los niveles así podrían aportar con sugerencias o empoderarse del trabajo realizado. Transparentar el número de pacientes en lista de espera y el número efectivo de pacientes resueltos, podría motivar al equipo de salud a realizar un trabajo más armónico y eficiente. El jefe de pabellón debe ser un líder de servicio, capaz de dar motivaciones trascendentes a los funcionarios a su cargo, es decir, que se sienta parte del proceso para llegar a un objetivo común. Por tanto, hacer parte del problema y de la solución al grupo de trabajo es una excelente opción.

En relación al subproceso quirúrgico:

El Minsal refiere[6] que se inicia a partir de la emisión por parte de un médico de la solicitud de pabellón para un paciente que requiere de una intervención quirúrgica. Las etapas que componen este subproceso son:

- Priorización de solicitudes (interconsultas): Lograr el acceso igualitario para todos los pacientes, generando una propuesta de tabla quirúrgica que debe considerar aspectos del paciente, aspectos clínicos y características de los prestadores de salud. Considerar: tiempos de espera, patologías y condición del paciente, para poder tomar una decisión lo más real posible.

- Gestión de recursos: Velar por la disponibilidad de recursos necesarios para realizar el acto quirúrgico. Implica revisar la propuesta de tabla y luego contrastarla con los recursos disponibles para poder atender dichas solicitudes de cirugía.

- Preparación del paciente: La etapa de preparación de paciente consiste principalmente en contactar al paciente, para luego entregar las indicaciones prequirúrgicas, acogerlo, llevar a cabo la evaluación preanestésica y, finalmente, una vez realizado lo anterior al trasladarlo al pabellón en el cual será intervenido.

- Preparación de pabellón: Chequear que el pabellón contemple las condiciones necesarias y esté en óptimas condiciones para poder realizar la intervención quirúrgica. En el caso de que algunos de estos puntos presente problemas, se deberá gestionar la solución en forma inmediata, de no ser posible la corrección del problema se informa al equipo quirúrgico y se da término por completo al proceso quirúrgico.

- Registro de Prestaciones e insumos: Registro en Ficha clínica y formularios asociados de todas las prestaciones otorgadas al paciente y del detalle de insumos utilizados para su atención.

- Intervención quirúrgica: El proceso clínico correspondiente 
al conjunto de actividades secuencialmente ejecutadas por el equipo quirúrgico o personal de pabellón. Considera la recepción del paciente en el pabellón, la ejecución de la primera y segunda pausa quirúrgica, la administración de anestesia al paciente y la realización de la intervención quirúrgica propiamente tal. Luego del acto quirúrgico se lleva a cabo la tercera pausa quirúrgica, para finalizar la intervención, se procede indicar y gestionar el traslado del paciente a cargo del médico anestesista.

En este subproceso, la optimización no puede estar orientada a disminuir tiempos de anestesia o cirugía, dado que podría disminuir la calidad y seguridad de la atención brindada. Por tanto, el enfoque debe estar en mejorar los tiempos entre las intervenciones, potenciar la puntualidad y no retrasar el comienzo de las cirugías, sobre todo la primera.

Nuestras recomendaciones para esta etapa son: tener personal de apoyo en hora punta y de colación para poder ejecutar relevos, aumentar cirugías ambulatorias para disminuir estancia media hospitalaria, paquetizar los insumos para cada tipo de cirugía, dar incentivos monetarios a todo el personal para disminuir ausentismo y retrasos, además de mejorar la ocupación de los pabellones. Varias de estas medidas ya se realizan en el sistema privado, pero no es una realidad en el servicio público. Para implementarlas falta aumentar la dotación de cargos, aumentar la glosa presupuestaria, etc.

En relación al subproceso posquirúrgico:

El Minsal[6] dice que este punto considera la recuperación inmediata de la anestesia poscirugía hasta su traslado a sala de hospitalización para cuidados críticos, medios o básicos según requiera o al domicilio como alta.
En este punto es importante para poder asegurar una atención de calidad y segura, es que exista un profesional médico y/o de enfermería con labores exclusivas de este proceso, lo que no es una realidad en todo nuestro país.

\section{Conclusión}

A lo largo de este documento se ha repetido en múltiples instancias la relevancia del trabajo multidisciplinario y queremos recalcar además, que es necesario que se trabaje en conjunto con todos los servicios clínicos que participan en el proceso quirúrgico. Actualmente, las instituciones trabajan siendo administradas cada una en forma separada, sobre todo cuando hablamos del trabajo administrativo y del clínico. Esto causa problemas en la planificación, lo que lleva a una gestión de contingencia y no de eficiencia de recursos[2].

Es fundamental que las jefaturas de servicios quirúrgicos no sólo tengan conocimientos clínicos, sino que de gestión y administración, para así hacer más eficiente la utilización de recursos y con ello, aumentar la productividad de pabellón.

Creemos que lo esencial para poder tener mejoras significativas en una estructura tan rígida como es el pabellón, es lograr volverla un ente dinámico en que participan múltiples agentes, donde cada parte es fundamental e irreemplazable, pero con el enfoque en dar atención de calidad y segura a los pacientes. Si agregamos a eso una estandarización y unificación de criterios, junto a jefes de servicio con amplios conocimientos clínicos y de gestión, tendremos pabellones con una mejor utilización de recursos para poder lograr satisfacer las necesidades de la población.

\section{Anexo 1}

Definiciones descritas por el ministerio de salud[4]:

1) Quirófano: Es el espacio físico, de acceso restringido, con autorización sanitaria para su funcionamiento, que cuenta con instalaciones, equipamiento, instrumental y los elementos de uso quirúrgico necesarios y suficientes para realizar una intervención quirúrgica mayor en condiciones de asepsia y seguridad.

2) Horarios de funcionamiento de los quirófanos, los cuales se dividen en:

- Horario hábil: Es el horario que comprende la jornada diaria de trabajo, de 08:45 h por día, de lunes a viernes generalmente en el horario comprendido entre las 08:00 y las 17:00.

- Horario inhábil de lunes a viernes: Es el horario posterior al término del horario hábil generalmente comienza a las 17 h y termina a las 08:00 del día siguiente.

- Horario Inhábil de sábado, domingo y festivos: Se define como el total de horas utilizadas en los quirófanos en trabajo, durante los días sábado, domingo y festivos.

3) Números de quirófanos en dotación: Corresponde a la cantidad de quirófanos asignados al establecimiento por la autoridad competente, instalados y dispuestos para realizar cirugía mayor (informados por resolución) y que funcionan regularmente. El número de quirófanos en dotación no es modificable por fluctuaciones temporales.

4) Promedio mensual de quirófanos habilitados: Corresponde al promedio mensual de quirófanos habilitados que se encuentran en condiciones de uso para realizar cirugía mayor, esto es que cuente con infraestructura y el equipamiento necesario para funcionar y que cumpla con la Norma Técnica Básica (NTB).

5) Promedio mensual de quirófanos en trabajo: Corresponde al promedio mensual de quirófanos disponibles y habilitados para ser utilizados en cirugía mayor, que cumplen con las NTB y además cuenta con la dotación de recursos humanos necesarios para funcionar.

6) Total, de horas mensuales de quirófanos habilitados: Corresponde a las horas mensuales de los quirófanos habilitados, que se encuentran en condiciones de funcionamiento en horario hábil. Cada quirófano debe estar disponible para cirugía mayor electiva a lo menos ocho horas diarias de lunes a viernes, descontado 45 min de colación respecto a las 44 h contratadas semanales, es decir, el total de horas habilitadas mensuales es de $8 \mathrm{~h}$ por el total de días hábiles del período y por el número de quirófanos habilitados. 
7) Total de horas mensuales de quirófanos en trabajo: Se refiere al total de horas mensuales disponibles de los quirófanos en trabajo; es decir, que se encuentran en condiciones de uso para realizar cirugía mayor electiva. Considera los factores recurso humano, equipamiento y cambios estacionales.

8) Horas mensuales programadas de tabla quirúrgica de quirófanos en trabajo: Corresponde a las horas quirúrgicas que se proyectan ocupar según la disponibilidad de los quirófanos en trabajo. Se desagrega según programación para beneficiarios en modalidad institucional, modalidad libre elección y otros. La información es proporcionada por el equipo del área de pabellones.

9) Horas mensuales ocupadas de quirófanos en trabajo en horario hábil: Corresponde registrar el total de horas utilizadas en los quirófanos en trabajo. Éstas corresponden a las horas ocupadas por las intervenciones quirúrgicas más las horas de preparación. Las horas ocupadas se deben calcular en base a las horas reales consignadas en los registros de quirófanos, no realizar aproximaciones. Esta información se desagrega según ocupación por beneficiarios en modalidad institucional, modalidad libre elección y otros. Esta información es proporcionada por el equipo del área de pabellones.

10) Horas mensuales ocupadas de quirófanos en trabajo horario inhábil de lunes a viernes: Corresponde registrar el total de horas utilizadas en los quirófanos en trabajo en horario inhábil. Éstas corresponden a las horas ocupadas por las intervenciones quirúrgicas más las horas de preparación. Las horas ocupadas se deben calcular en base a las horas reales consignadas en los registros de quirófanos, no realizar aproximaciones. Esta información se desagrega según ocupación por beneficiarios en modalidad institucional, modalidad libre elección y otros. Esta información es proporcionada por el equipo del área de pabellones.

11) Horas mensuales ocupadas de quirófanos en trabajo los días: sábado, domingo y festivos: Corresponde registrar el total de horas utilizadas en los quirófanos en trabajo, los días sábado, domingo y festivos. Éstas corresponden a las horas ocupadas por las intervenciones quirúrgicas más las horas de preparación. Las horas ocupadas se deben calcular en base a las horas reales consignadas en los registros de quirófanos, no realizar aproximaciones.

12) Total de horas mensuales ocupadas de quirófanos en trabajo cirugía menor y otros procedimientos: Corresponde registrar el total de cirugías menores y otros procedimientos, realizados en los quirófanos, independiente del horario y día en que se realicen. Esta información se desagrega según ocupación por beneficiarios en modalidad institucional, modalidad libre elección y otros. Esta información es proporcionada por el equipo de pabellón.

13) Horas de preparación: Se registra el número de horas ocupadas en la preparación del quirófano para una próxima cirugía (desinfección concurrente y terminal, limpieza de instrumental, etc.). Este registro es responsabilidad de la unidad de pabellón y deberá ser entregado a la unidad de estadística en el plazo establecido.

14) Días de estada prequirúrgicos: Corresponde al número de días transcurridos entre la fecha de ingreso y la fecha de la intervención quirúrgica. Se refiere a los días promedio de permanencia de las personas antes de ser intervenidos quirúrgicamente. El objetivo es evaluar la utilización que se le da a la cama, con él se puede evaluar la prolongación innecesaria de los días de hospitalización, descoordinación entre los servicios administrativos, diagnósticos o terapéuticos y las unidades funcionales o unidades de hospitalización.

15) Pacientes intervenidos: Se refiere a todo paciente que ha sido sometido a una intervención quirúrgica mayor, ajustándose a la definición descrita en el REM BS, para este tipo de intervención.

16) Programación tabla quirúrgica: Es el proceso de asignación de tiempo y espacio en un quirófano a un paciente, para la realización de una intervención quirúrgica mayor. Su mejoramiento incide directamente en la eficiencia de las horas disponibles en quirófano y por cierto disminuye el indicador de suspensión. Es clave para asegurar los recursos materiales y humanos que permitan sostener la tabla y así mismo garantizar la oportunidad y calidad de la atención de las personas priorizadas y preparadas.

17) Suspensión: Todo evento en el cual la persona que estando en la tabla quirúrgica publicada, no es intervenida, independiente de la causa de suspensión (de la persona, quirófano, unidades de apoyo, equipos quirúrgicos). El objetivo de este ítem es disminuir el número de intervenciones suspendidas por cada especialidad quirúrgica (de acuerdo a la cartera de servicios del establecimiento). Considerando que la meta de suspensiones es llegar al 7\%. Los gestores de quirófano deberán en conjunto con los cirujanos y anestesistas, programar las intervenciones con el objeto de disminuir las suspensiones.

18) Paciente condicional: Todo paciente en tabla quirúrgica definitiva, que queda en condición de suplencia frente alguna suspensión de paciente de cirugía programada. Esto no significa, necesariamente, que vaya hacer intervenido quirúrgicamente el día citado.

Debe cumplir los siguientes criterios:

- Contar con el consentimiento del paciente o tutor legal.

- No incluir aquellos pacientes que se vean afectados por el ayuno, preparaciones especiales, enfermedades de salud mental, aspectos especiales (adulto mayor, ruralidad, dependiente de cuidados).

- No incluir pacientes de menos de 6 meses de edad.

- No incluir pacientes que requieran cirugías prolongadas y/o complejas.

Consideraciones relevantes:

- No se consideran como suspensiones los pacientes que son incorporados como condicionales a la tabla quirúrgica y posteriormente no son realizados.

- Se debe contar con criterios de incorporación a tabla quirúrgica programada de pacientes condicionales que no fueron intervenidos, esto debe considerar dejar solo por una vez al paciente en forma condicional, sino ha sido intervenido en esa oportunidad, se debe asurar su incorporación en la tabla quirúrgica en oportunidad próxima e informarle al paciente.

19) Días de estada prequirúrgicos: Corresponde registrar el promedio de días prequirúrgicos por especialidad y por rango etarios, independiente de la previsión y modalidad de atención (MAI, MLE y No beneficiarios). No se incluyen las cirugías ambulatorias 
en este registro, ya que no generan días de estada prequirúrgicos y no hacen uso del recurso cama hospitalaria (dotación).

20) Pacientes intervenidos: Corresponde registrar el número de pacientes intervenidos quirúrgicamente en el mes del informe, según la especialidad por la que fue intervenido y por grupo etario. Se registra el número total de cirugías programadas, en los diferentes horarios (hábil, inhábil y fines de semana) e independiente de la previsión y modalidad de atención (MAI, MLE y No Beneficiario). Se incluyen, además, las cirugías ambulatorias electivas.

21) Número de pacientes suspendidos: Corresponde registrar el número de pacientes suspendidos, definida como a toda persona que se encontraba programada en la tabla por especialidad y grupo etario. Se deben registrar todas las intervenciones quirúrgicas electivas, suspendidas, en los diferentes horarios (hábil, inhábil y fines de semana), independiente de la previsión y modalidad de atención institucional, libre elección y pacientes no beneficiarios FONASA. En esta sección, las variables se registran en base a las diferentes especialidades del arancel FONASA y el registro es responsabilidad de la unidad de pabellón (la fuente de datos es la tabla quirúrgica) y debe ser entregado a la unidad de estadística en el plazo establecido.

22) Intervenciones quirúrgicas mayores no ambulatorias electivas: Corresponde a una intervención quirúrgica que implica la entrada a cavidades normalmente estériles como peritoneo, pleura y/o cavidad ocular, que conlleva un mayor riesgo de sangrado y que requiere de sedación profunda, anestesia general o anestesia regional y que por el tipo de diagnóstico y las características clínicas del paciente, su realización puede ser diferida en el tiempo.

23) Intervenciones quirúrgicas mayores ambulatorias electiva: Corresponde a una intervención quirúrgica mayor, que de acuerdo al diagnóstico y condición clínica de la persona, puede ser electiva. Se realiza en quirófano ambulatorio o central y la recuperación del paciente es menor a $24 \mathrm{~h}$. El período de observación posoperatoria debe ser realizado en una unidad destinada a este fin y no en una cama de dotación.

24) Intervenciones quirúrgicas mayores ambulatorias urgencia: Corresponde a una intervención quirúrgica mayor, que de acuerdo al diagnóstico y condición clínica de la persona, debe ser de urgencia. Se realiza en quirófano ambulatorio o central y la recuperación del paciente es menor a $24 \mathrm{~h}$. El período de observación posoperatoria debe ser realizado en una unidad destinada a este fin y no en una cama de dotación.

25) Intervenciones quirúrgicas mayores no ambulatorias urgencia: Corresponde a una intervención quirúrgica mayor, que de acuerdo al diagnóstico y condición clínica de la persona, debe ser de urgencia. Se realiza en quirófano y la recuperación del paciente es realizado en una cama hospitalaria de dotación.

26) Cirugía menor: Corresponde a una intervención quirúrgica diagnóstica y/o terapéutica de baja complejidad y riesgo vital, con escasas complicaciones, realizada bajo anestesia local y mínima medicación preoperatoria.

27) Procedimientos clínicos: Corresponde a prestaciones de salud, que se otorgan a un paciente para efectos diagnósticos y/o terapéuticos, que implican el uso de equipamiento, instrumental, instalaciones y profesionales especializados, dependiendo de la complejidad del procedimiento y de las condiciones clínicas del paciente.

28) Amputación por pie diabético: Se refiere a la intervención quirúrgica de amputación, en las extremidades inferiores de un paciente, con diagnóstico de diabetes mellitus, con complicaciones circulatorias periféricas (pie diabético), de acuerdo a los siguientes niveles de amputación:

- Ortejos: Se refiere a la amputación de uno o más ortejos, y a nivel de metatarsos.

- Pie completo: Ésta se refiere a la amputación infra o supracondílea. Las definiciones conceptuales de las otras variables incluidas en esta sección ya fueron definidas anteriormente.

29) Causales de suspensión:

1. Atribuidas al paciente

- No se presenta/no se ubica.

- Rechaza operación.

- Patología aguda.

- Patología crónica descompensada.

- Falta de ayuno.

- Sin suspensión de anticoagulante u otras drogas proscritas (excluidas).

- Atraso en el ingreso.

- Patología no informada, no conocida (alergia al látex).

- Estudio incompleto.

- Exámenes alterados no corregidos.

- Falta de preparación de piel, intestinal, antibiótica u otra específica.

- Sin evaluación de especialista indicada.

- Descompensación en pabellón.

- Sin indicación quirúrgica.

- Anticipación de cirugía por agudización de patología.

- Paciente fallece.

2. Administrativas

- Error de programación.

- Reemplazado por urgencia.

- Sin cupo en recuperación.

- Falta disponibilidad de cama en unidades de paciente crítico. 
- Documentación incompleta.

- Sin consentimiento informado firmado/ausencia de tutor legal consignado.

3. Unidades de apoyo clínico

- Instrumental y/o material con falla de esterilización.

- Instrumental incompleto o no disponible.

- Equipamiento no operativo.

- Falta sangre o hemoderivados.

- Falta medicamentos/stock insuficiente.

- Falta de insumos/stock insuficiente.

- Falta de ropa quirúrgica/stock insuficiente.

- Falla coordinación con unidad de imagenología.

- Falla coordinación con unidad anatomía patología (biopsia rápida).

\section{Equipo quirúrgico}

- Falta/disponibilidad de cirujano.

- Falta/disponibilidad de técnico paramédico.

- Falta/disponibilidad de anestesiólogo.

- Falta/disponibilidad profesional no médico.

- Prolongación de tabla.

\section{Infraestructura}

- Falta de personal de aseo.

- Falla energía eléctrica.

- Falla de climatización.

- Falla de red húmeda.

- Falla ascensor.

- Falla gases clínicos.

\section{Emergencias}

- Desastres naturales: Terremotos, aluviones, tsunamis, inundaciones, erupciones volcánicas, etc.

- Destrucción repentina e irrecuperable de la infraestructura del hospital.

- Desastres de eventos deportivos.

- Accidentes múltiples.

- Incendios forestales.

- Incendios o amago de incendio.

- Inundaciones.

- Derrame de productos químicos.

- Aviso de bombas.

- Actos delictuales.

- Actos de marcada connotación pública.

- Emergencias sanitarias.

\section{Gremiales}

- Paro o movilización de funcionarios. 
Artículo de Reflexión

Anexo 2.

Tríptico resumen para difusión de Protocolo de Evaluación Preoperatoria

\begin{tabular}{|c|c|}
\hline \multirow{2}{*}{\multicolumn{2}{|c|}{$\begin{array}{c}\text { ¿QUÉ MEDICAMENTOS } \\
\text { DEBO SUSPENDER? }\end{array}$}} \\
\hline & \\
\hline Medicamento & $\begin{array}{l}\text { Tiempo de } \\
\text { suspensión previo a } \\
\text { cirugia }\end{array}$ \\
\hline \multicolumn{2}{|l|}{ Cilostazol } \\
\hline \multicolumn{2}{|l|}{ IECAYARA III } \\
\hline \multicolumn{2}{|l|}{$\begin{array}{l}\text { B bloqueadores y bloqueadores de canales de } \\
\text { calcio }\end{array}$} \\
\hline \multicolumn{2}{|l|}{$\begin{array}{l}\text { Estatinas, antiepilépticos, levotiroxina, } \\
\text { aspirina, benzodiacepinas, } \\
\text { antiparkinsonianos, inhaladores }\end{array}$} \\
\hline \multicolumn{2}{|l|}{ Anticoagulantes dicumarinicos } \\
\hline \multicolumn{2}{|l|}{ Diuréticos } \\
\hline \multicolumn{2}{|l|}{$\begin{array}{l}\text { Antiagregantes (clopidogrel, plasugrel, } \\
\text { ticagrelor) }\end{array}$} \\
\hline \multicolumn{2}{|l|}{ Dalteparina / henoxaparina } \\
\hline \multicolumn{2}{|l|}{ Heparina no fraccionada } \\
\hline \multicolumn{2}{|l|}{ Metformina y glibenclamida } \\
\hline Insulina NPH & \\
\hline
\end{tabular}

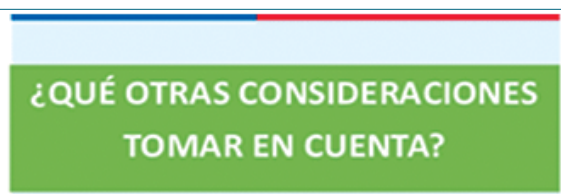

Exámenes: válidos hasta 6 meses con condiciones clínicas estables. De no ser así, tomar exámenes actualizados. Ojo: electrolitos en IRC y pruebas de coagulación en pacientes en TACO requieren control el día de la cirugía.

Ecocardiograma: antecedente de insuficiencia cardiaca, cardiopatía isquémica (angina o IAM), capacidad funcional < $4 \mathrm{MET}$, arritmias, valvulopatias, capacidad funcional no evaluable.

ECG: Pacientes con angina o IAM, insuficiencia cardiaca, IRC, AVE o TIA, EAO, mayores de 65 años, diabéticos, hipertensos, antecedente de arritmias, usuario marcapasos o DAl, consumo de cocaína, valvulopatías moderadas o severas.
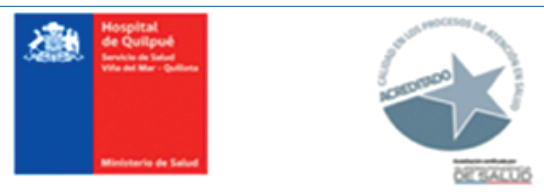

INSTRUCTIVO DE EVALUACIÓN

PREQUIRÚRGICA PARA

CIRUGÍA ELECTIVA

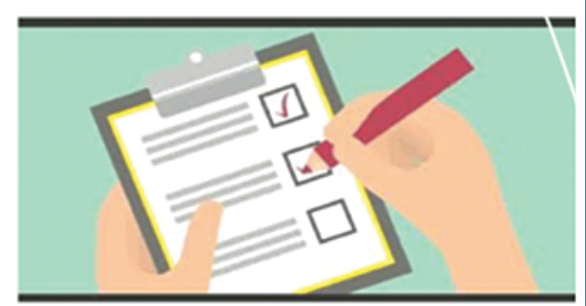

Espirometría: Antecedente de patología pulmonar, con historia de neumonectomía o lobectomía, paciente con riesgo elevado de compromiso de función pulmonar intraoperatorio (obesos, SAHOS).

\section{Referencias}

1. Uso Eficiente de Quirófanos Electivos y Gestión de Lista de Espera Quirúrgica No GES.

2. Wolff Rojas PA. Optimización de los procesos de gestión de pabellones quirúrgicos en hospitales públicos. 2012.

3. Albareda J, Clavel D, Mahulea C, Blanco N, Ezquerra L, Gómez J, et al. ¿Realizamos bien la programación quirúrgica? ¿Cómo podemos mejorarla? Revista Española de Cirugía Ortopédica y Traumatología. Noviembre de 2017;61(6):375-82.

4. Ministerio de Salud - Gobierno de Chile. Manual Series REM 2020. 2020.

5. Marjamaa R, Vakkuri A, Kirvelä O. Operating room management: why, how and by whom?: OR management. Acta Anaesthesiologica Scandinavica. Mayo de 2008;52(5):596-600.

6. MINSAL. Procesos de administración de sucursales [Internet]. [citado 20 de febrero de 2021]. Disponible en: https://web.minsal.cl/ sites/default/files/files/6_\%20Proceso\%20Quir\%C3\%bargico\%20 2015\%20v_2.doc

7. Subsecretaría de Redes Asistenciales M. Subproceso Prequirúrgico. 2018.

8. Jansson A, Delgado C. Optimización del proceso de cirugía en hospitales públicos. Una aplicación de la modelacion matemática entera en la prestación de atención quirúrgicas en el hospital del Salvador, Santiago de Chile. Pharos [Internet]. Mayo de 2000;7(1). Disponible en: https://www.redalyc.org/articulo.oa?ld=20807104 